\title{
EWA PIROGOWSKA
}

Université Adam Mickiewicz

\section{DE LA MÉTAPHORE DANS LA LANGUE INFORMATIQUE}

\section{Le repérage des métaphores qui motivent une terminologie est une façon de rendre compte de la dimension culkurelle} des sciences et des techniques

(Kathryn English, 1998)

\begin{abstract}
Pirogowska Ewa, De la métaphore dans la langue informatique [On metaphor in the language of information science]. Studia Romanica Posnaniensia, Adam Mickiewicz University Press, Poznań, vol. XXX: 2003, pp. I3I-141. ISBN 83-232-1270-8. ISSN 0137-2475.
\end{abstract}

The French of information science is a variant of a lively general language submitted to constant changes. In order to bring closer, better understand and get acquainted with the technical terms either names of equipment, programmes, or situations peculiar to computer reality, the user refers to everyday Jife (e.g. passerelle, English gateway; souris, English mouse) or to universal or mythological concepts (c.g. argonautes - internautes) thanks to the phenomenon of metaphor. It sometimes happens that the original concept, which served as a basis for the creation of a metaphor, is obliterated memory and computer users apply a new term being unaware of its origin. Just in this way during metaphoric operations totally new words are formed and they influence the specific character of the language of information science.

La métaphore dans les langues de spécialité où se situe également la langue informatique, sert à transmettre les informations inconnues ou difficiles à l'aide des images concrètes et faciles (Kacprzak 2000: 184). La métaphore est donc un procédé de langue qui consiste à employer un terme dans un contexte spécifique par substitution analogique, sans qu'il y ait d'élément qui introduit formellement une comparaison. Cette définition empruntée au Petit Robert nous montre qu'à l'origine d'une métaphore se trouve une comparaison. La souris, nom du dispositif aidant à gérer l'écran de l'ordinateur, est fondé sémantiquement par le nom du petit animal dit souris. D'autre part, les jeunes pratiquent le surf tant sur les vagues de mer que de cyberespace. Nous comparons nos ordinateurs aux gens avec le degré d'intelligence. Il y a de la métaphore partout!

C'est la métaphore qui, pour la plupart des cas, est à l'origine des sens nouveaux des mots. Elle enrichit le vocabulaire (surtout dans la situation de l'emprunt 
continu de la langue anglaise) non seulement pour trouver des substituts aux emprunts, mais aussi pour désigner des réalités nouvelles.

Comme nous le savons, les mots nécessaires à la communication peuvent être des néologismes de forme et de sens ou de sens seulement. On les forme dans le domaine étudié, grâce, du moins partiellement, aux expressions métaphoriques venues à l'esprit de créateurs des logiciels et du matériel informatique. Ces termes sont inventées par les informaticiens et stockés par ce langage spécifique de l'informatique.

Elisabeth Eek souligne le rôle de la métaphore dans le domaine: «Depuis les extensions de sens jusqu'aux néologismes en passant par les nombreux calques littéraux, l'on se rend compte que le stock lexical français se prête, par transparence symbolique, à l'élaboration d'une terminologie moderne» (Eek 1998: 2). La métaphore contribue sensiblement à l'enrichissement de la terminologie informatique par sa capacité de l'extension du sens. L'auteur du présent article voudrait étudier le phénomène en question à l'exemple du vocabulaire informatique, sur des exemples dont les définitions sont enregistrées dans dictionnaires techniques choisis (vide la bibliographie). Notre liste a été complétée par les emprunts faits à la publication de Gabriel Otman Les mots de la cyberculture d'où nous nous permettons de puiser à plusieurs reprises. Nous présenterons la problématique étudiée repartie en plusieurs champs sémantiques.

\section{CHAMPS SEMANTIQUE ANTHROPOMORPHIQUE: HOMME}

\section{VEUVE (ang. widow)}

Le terme veuve, calque directe de "widow», désigne, dans le langage familier, épouse abandonnée (sur le plan des sentiments) au profit de l'ordinateur, actuellement le plus souvent soit pour jouer aux jeux vidéo soit pour surfer dans l'Internet (game widow, Net widow). Il serait fort justifié de parler aussi de veuf, comme ce sont souvent les maris qui doivent payer les factures élevées puisque leurs femmes ont aimé le bavardage en IRC.

\section{INTELLIGENCE, MÉMOIRE}

Les deux termes susmentionnés qui représentent le champ sémantique lié aux capacités mentales de l'homme, deviennent une base de la métaphore - intelligence artificielle. Elle est fondée sur l'observation simplifiée des processus de la compréhension, de la perception et de la résolution des problèmes qui se passent dans le cerveau humain. On enregistre également d'autres métaphores du même ordre ou l'on attribue les propriétés de l'homme à la machine: réseau intelligent, stylo intelligent, mais aussi machine à laver intelligente et lessive intelligente. Nous observons le même quant au terme mémoire. Nous nous permettons ici de raconter en quelques mots l'histoire de cette métaphore dite anthropomorphique, en profitant 
des informations provenant des Mots de la cyberculture (Otman 1998: 224-227). Charles Babbage, auteur d'une machine analytique (présentée en 1840!), considérée comme premier ordinateur, était contemporain de Mary Shelley, auteur de Frankenstein, première «machine humaine» ou «homme artificiel». Cette analogie entre l'homme et l'ordinateur a été développé par Alan Turing en XX siècle - il essayait de créer une machine «cerveau artificiel» en formulant aussi des termes comme: machine pensante, intelligence des machines, cerveau électronique qui ont donné naissance aussi à l'intelligence artificielle. La mémoire est, avec l'unité centrale, l'élément essentiel de l'ordinateur - elle lui permet de stocker les informations (sous forme binaire) en conservant les valeurs numériques et des instructions de calcul. Il faudrait dire encore qu'il existe deux sortes de mémoire, mémoire vive («random access memory») et mémoire morte («read-only memory»), créations autonomes de la langue française et bons exemples des métaphores «du vivant».

\section{NEZ ARTIFICIEL; ÉLECTRONIQUE}

La métaphore de l'organe humain est ici très claire. Elle désigne un dispositif capable de capter les odeurs en réalisant leurs empreintes numériques, ainsi qu'il peut servir de système de sécurité et de contrôle d'accès à des systèmes sensibles (Otman 1998: 267).

\section{BUREAU (ang. desktop)}

Nous connaissons tous cette simple métaphore, mais pour être précise nous citons ici la définition: «espace de travail constitué par l'écran de l'ordinateur sur lequel se trouvent des icônes représentant des applications ou des outils de bureau» (Otman 1998: 48). Poúr comprendre d'où nous vient cette métaphore, regardons un peu l'histoire du mot bureau. Au XIII ${ }^{\mathfrak{e}}$ s. les moines copiaient les livres en déposant sur leurs tables de travail des carrés d'étoffe de bure (la même qui servait aux habits) - le bureau signifiait alors cette couverture de table. Ensuite le sens du mot a évolué - le bureau signifiait la table elle-même, la pièce où se trouvait ce meuble et, surtout actuellement, tout l'immeuble avec des dizaines de pièces-bureaux avec un meuble-bureau. La métaphore dans le langage informatique, donc celle de l'espace sur l'écran où l'on organise son travail et projets, est encore renforcée par l'utilisation dans le même contexte des métaphores comme dossier, fichier, document, gomme, ciseaux, presse-papiers, papier peint, corbeille, poste de travail (à consulter: Otman, Les mots de la cyberculture). Une des grandes innovations des créateurs de logiciels c'était de lier ce qui se passait à l'écran avec le corps de l'usager à l'aide du souris. On a saisi facilement une autre métaphore, délicate, mais bien visible: «la petite flèche contrôlée par la souris c'était notre doigt dans l'écran nous permettant de manipuler des dossiers virtuels» (voir: site http://martine.gingras.net).

Pour finir sur ce point de parler du bureau, nous voudrions ajouter la constatation de Denis Poussart (cité par Haman, 1996) que l'utilisation de l'ordinateur «converge vers une disparition des frontières physiques, est une démarche 
incontournable vers l'abstrait. Aujourd'hui, lorsque nous ouvrons notre ordinateur, ce qui apparaît à l'écran est une métaphore de notre bureau. Demain ce sera une métaphore de la planète».

\section{COURRIER ÉLECTRONIQUE (ang. e-mail)}

Aujourd'hui le réseau informatique sert aussi à l'échange des messages et des fichiers de données à l'image du courrier ordinaire où les lettres sont livrés en trains, en camions et enfin apportées par le facteur. Mais le courrier électronique est le plus rapide et, probablement, le plus sûr, il devient de plus en plus universel. Nous pouvons donc voir ici la métaphore de tout le phénomène qu'il est le courrier en général; les chevaux stationnant au relais de poste deviennent aujourd'hui modem, câbles de téléphone, logiciel de type Outlook Express; une adresse écrite main c'est simplement l'inscription comme abc@net.com. Il serait utile d'ajouter que le néologisme, un mot-valise courriel (courrier + électronique) est de plus en plus fréquent et désigne une adresse $e$-mail ou bien mél.

\section{COOKIE}

Un cookie c'est «un petit programme qui vient de s'insérer automatiquement et à l'insu de son utilisateur sur son disque dur à la suite d'une consultation Internet et qui permet de suivre son parcours Web à la trace» (Otman, 1998: 90). Comme le soulignent l'auteur cité et les créateurs de la série de manuels pour les débutants en informatique (Levine 1998: 16-17 et 132), un cookie sert à conserver les traces d'un utilisateur, mais il peut collecter seulement les informations données par lui-même. Le mot signifie «un petit gâteau sec», mais son emploi en informatique vient probablement du sens argotique. «Smart cookie» c'est un petit malin, «a hot cookie» c'est une jolie fille; tout le monde connaît «cookie monster» (dans «Sesame street») qui, comme un terme à part, est employé en informatique pour désigner un programme de piratage d'accès à un serveur. On observe en français un terme métaphorique très intéressant mouchard électronique (mouchard - espion, indicateur de police, dénonciateur).

\section{SERVEUR - CLIENT}

Le serveur s'occupe du stockage de programmes et de données, il distribue ensuite ces éléments à la demande des utilisateurs; c'est un logiciel et l'ordinateur en même temps. «Tout le réseau informatique requiert la présence d'un serveur qui sert de pilote au réseau» (Otman 1998: 348). Donc l'expression serveur-client ou client-serveur (calque de l'anglais) dessine l'image du réseau ou les clientsulilisateurs et le serveur-ordinateur où les données ont été stockées communiquent entre eux à l'aide de logiciels d'interrogation. La signification de la métaphore est simple - imaginons la scène dans un restaurant où le maître d'hôtel (garçon, serveuse, plus rarement serveur) nous sert des plats; il s'agit du même mécanisme. 


\section{MÉTAPHORES SUR L'INTERNET}

point com (.com)

Dans un monde qui se transforme à une vitesse inouïe en une «communauté.com» on ne reconnaît plus toutes les métaphores provenant d'idées transposées de la vie quotidienne en vie artificielle, celle de l'ordinateur, parce que cette dernière est déjà devenue quotidienneté. Point com (.com) est une métaphore pour l'informatique sur l'Internet, standardisée et ouverte, mais ses implications vont bien au-delà d'un site Web. Cette parallèle entre la réalité d'Internet et le besoin d'être toujours branché montre le niveau de notre dépendance du réseau, de la toile d'araignée mondiale où ubiquitous computing est un fait incontestable.

L'Internet c'est le réseau des réseaux d'ordinateurs interconnecté où l'information, découpée en "paquets» peut transiter par des milliers d'ordinateurs. Internet englobe notamment le Web, le courrier électronique et les groupes de discussion. Et le Web, à son tour, est appelé la toile d'araignée mondiale, filet tendu sur la planète, dont chaque maille serait un ordinateur et chaque fil une ligne de téléphone.

\section{AUTOROUTES, INFOROUTES, LABYRINTHE}

Internet est souvent comparé à des autoroutes de l'information. Cette expression est liée à la métaphore portée par la notion de la ligne droite qui pendant longtemps désignait le progrès, et "c'est sans doute pour cela que le mot d'autoroute est venu à l'esprit lorsqu'il s'est agi de nommer les réseaux multimédias en gestation» (Attali 1995). Mais il semble que cette métaphore ne soit plus actuelle, le monde d'information étant aujourd'hui un grand labyrinthe où l'on est forcé à chercher et à choisir sans cesse. L'informatique, terme désignant tout d'abord la science de l'information et non seulement le domaine réservé au hardware et software, se sert d'un support puissant, celui de l'Internet. Comme le réseau WWW se compose de mille chemins qui souvent se terminent en impasses, on parlerait plutôt des labyrinthes de l'information dans ce contexte-ci. A vrai dire toute l'informatique est labyrinthique: le microprocesseur est comme un labyrinthe des puces; la succession d'instruction binaires des programmes informatiques doit être lue comme une succession de choix d'emprunter tel ou tel chemin.

Dans cet univers, les mythes auront beaucoup à dire; et d'abord évidemment celui du Crétois qui fit du labyrinthe le lieu de dissimulation de la barbarie. Qui sera Minos, le pouvoir qui veut enfouir ses secrets dans le labyrinthe? Qui sera Thésée, qui veut les dévoiler? Et Ariane, la rebelle, qui lui donne le fil pour l'amour de la vie? Et Dédale, le génial inventeur du piège, seul capable de le déjouer? Et le Minotaure, la chimère, le monstre, l'inconscient, l'ennemi enfoui dans chaque homme, qu'il faut mettre au jour pour le détruire? Et Icare, le démiurge, qui, pour s'évader du labyrinthe, utilise les ailes mises au point par son père, le sage Dédale, mais qui montera trop haut et chutera? Que sera enfin la cire, grandeur et limite de l'intelligence humaine, parce qu'elle lui permet de fixer des ailes à ses épaules et 
d'échapper au labyrinthe par le haut, au moins aussi longtemps qu'il ne tente pas de s'approcher trop du soleil? De Dédale à Internet. Très loin. Et très proche. Exactement comme deux points voisins d'un labyrinthe (Attalj 1995).

\section{UBIQUITOUS COMPUTING}

Ubiquitous computing, pervasive computing (tous les deux de l'ang.: informatique qui pénètre, s'infiltre partout), objets communicants, agents intelligents, informatique diffuse, informatique embarquée - en voilà les désignations, avec une ombre métaphorique, de la réalité de nos jours, celle «de ce fameux troisième âge de l'informatique où elle disparaît puisqu'elle est partout» (site Fondation Internet Nouvelle Génération, 2002). La notion de ubiquitous computing a été développé par Marc Weiser dès l'an 1996 dans la situation où le réseau des réseaux s'est déjà parfaitement intégré dans la vie de l'homme. Cette idée s'opposait à la notion de la réalité virtuelle qui, selon Weiser, tente de mettre l'homme dans l'ordinateur. En plus, ubiquitous computing nous fait laisser de côté la métaphore du bureau comme porte d'entrée du monde de l'information. Il faudrait préciser maintenant pourquoi nous parlons du troisième âge de l'informatique. La première époque était quand plusieurs personnes étaient connectées à un seul ordinateur. Ensuite nous avons eu le temps de l'ordinateur personnel, donc le temps de l'interaction entre une personne et sa propre machine intelligente. Mais l'Internet a modifié totalement ces relations «en créant, avec son architecture souple et ses standards, un espace de communication pour les individus mais aussi, potentiellement, pour des objets. (...) l'idée d'agir sur les objets à travers l'Internet, et même de permettre aux objets de se coordonner entre eux, d'interagir, est apparue» (Menga 2002). Nous pourrions créer ici plusieurs idées de la métaphore - pourquoi pas même celle de trois âges de la vie humaine, l'âge d'or, d'argent et de fer? Il nous semble pourtant très intéressant de comparer l'informatique à un être omniprésent, à une âme, un esprit qui se trouve partout mais on ne le voit pas, on ne se rend pas compte même de son existence comme on ne réfléchit pas sur le fonctionnement des horloges mesurant le non-mésurable - le temps. L'informatique et ses moyens, dont le plus universel soit l'Internet, gère notre vie, le rend plus facile et plus rapide, mais en même temps elle nous rend dépendants et incapable de nous débrouiller sans elle. «(...) l'informatique se dilue dans le paysage à en devenir invisible; avec une notion d'invisibilité à plusieurs niveaux: l'invisibilité réelle parce que ça devient tout petit, et l'invisibilité mentale» (Menga 2002) parce que l'on ne perçoit plus son existence, diluée dans le paysage. L'informatique "pervasive», diffuse serait donc une métaphore de ... la magie?

\section{INTERNAUTES}

Il y eut d'abord les Argonautes, qui naviguèrent avec Jason à la recherche de la Toison d'or; puis les aéronautes qui défièrent les vents; enfin les astronautes qui décrochèrent la Lune. Voici le temps des internautes, dont le message tapé sur l'écran de leur ordinateur pcur atteindre en une fraction de seconde les coins les plus reculés de la Terre (Télérama, le 21 juin 1995). 
Comme l'explique l'auteur dont nous profitons beaucoup dans cet article, Gabriel Otman (1998: 181), l'appellation internaute, provenant de deux mots: Internet et astronaute, désigne l'utilisateur du réseau Internet qui, comme l'astronaute explorant l'espace, explore le cyberespace. On rencontre très souvent un autre terme: netsurfer qui nous renvoie à l'image d'un sportif pratiquant le surf ou la planche à voile, alors l'image très «cool» comme Internet l'est. Les internautes conquérants d'un nouvel espace, des navigateurs des cyber-navires; nous en reparlerons dans le sous-chapitre sur le champs sémantique de la route.

\section{VILLAGE GLOBAL}

Comme l'on peut lire sur le site de Archipress, dans l'article de Réta Benkirane, la métaphore du village global fut énoncée pour la première fois en 1962 par le canadien Marshall McLuhan pour décrire l'essor des médias électroniques et leur influence croissante sur les sociétés humaines. Depuis, «sa formule du village global a fait fortune pour être systématiquement couplée à la "globalisation" ou mondialisation». Chacun de nous vit dans ce village et est capable de communiquer en une seconde avec l'ami de n'importe quel but du monde, recevoir des informations et travailler. Ce fameux village planétaire a ses deux côtés: d'un côté, «on consacre la libre circulation des marchandises, les idées, l'argent défilent d'un continent à l'autre à la vitesse de la lumière; et de l'autre, les frontières se ferment aux hommes du Sud, des barricades administratives sophistiquées, dressées pour se prémunir d'hypothétiques remontées massives, bloquent au quotidien les petits passages multiples et saisonniers. De cette façon duale est programmée l'intégration des grands ensembles, riches et forts et la désintégration des pays moins nantis». C'est justement comme dans un petit village où il vaut mieux être riche et admiré plutôt que pauvre et rejeté; dans ce dernier cas il faudrait fuir la petite communauté locale. Mais est-ce que dans notre village global on a un choix?

\section{CHAMPS SÉMANTIQUE «ROUTE»}

\section{NAVIGATION, NAVIGATEUR (ang. to browse, browser), CYBERESPACE}

Ces métaphores sont devenues universelles et communes au point où l'on remarque à peine leur provenance maritime. Comme nous apprenons chez Otman (1998: 259), c'est au début du XX $X^{\mathfrak{e}}$ s. que l'aéronautique naissante a adopté plusieurs expressions de la terminologie maritime, parmi d'autres: pilote, navigateur, navigation. Actuellement l'espace à conquérir est un espace cybernétique, le cyberespace - métaphore désignant le domaine des interactions entre données et réseaux et accessible par ordinateurs, mais au sens large le monde global de tous les ordinateurs et systèmes connectés par Internet. Dans ce cyberespace naviguent des cyber-navigateurs, brouteurs (peut-être ceux qui, comme une chèvre au pré, 
cherchent ici et là en broutant les informations?), fureteurs (donc la comparaison à ceux qui chassent avec un furet), butineurs (à l'image des abeilles).

Il serait utile de dire que le suffixe - naute (astronaute, cosmonaute, spationaute, internaute, cybernaute, etc.) provient du grec - nautês ce qui signifie marin, navigateur. Les expressions comme naviguer sur Internet (en version plus «sportive» surfer) ou logiciel de navigation se sont vite implantés en français, surtout que le sens familier du verbe naviguer, tourner en rond en quête d'un objectif introuvable, est employé dans le contexte routier.

Il est intéressant de noter qu'aux Etats-Unis, parallèlement à l'expression «surfing the Internet» sévit une expression jugée peut-être moins compétitive et plus décontractée. II s'agit de l'expression «cruising the Internet» qui signifie littéralement "croiser, faire une croisière sur Internet». Elle exprime mieux les aspects flânerie, promenade et découverte d'un parcours sur le Web (Otman 1998: 260).

\section{PILOTE (ang. driver)}

Pilote c'est un programme grâce auquel les périphériques comme le clavier, le scanner ou l'imprimante peuvent fonctionner en association à un ordinateur. Le terme anglais «driver» signifie, dans le sens de base, le conducteur d'une voiture ou d'un train, cependant le calque métaphorique française pilote nous renvoie au champs sémantique de la navigation maritime ou aérienne. Ce terme est, d'ailleurs, antérieur à navigateur dans le domaine du réseau Internet.

\section{DISTANT (ang. «remote»)}

En général cet adjectif ajouté à un terme lui apporte le sens de celui qui ne se trouve pas dans le même lieu que l'utilisateur (p.ex. terminal distant, commande à distance), alors l'on comprend un tel épithète dans son extension physique. Mais nous avons pensé à une compréhension du mot - quand on parle d'une personne réservée, on l'appelle «distant, -e». Est-ce que les ordinateurs, photocopieuses, imprimantes ne nous semblent pas être parfois distants dans le sens des machines sans âme, impersonnelles et froides?

\section{PASSERELLE (ang. gateway)}

Ce terme constitue un exemple de la métaphore routière liée aux réseaux de télécommunications. On appelle passerelle chaque équipement matériel qui relie deux portions de réseaux distinctes et de type différent (p.ex. réseau local et Internet, voir aussi: Otman 1998: 289) et qui permet de communiquer entre eux. Il y des passerelles téléphonique, de courrier électronique, d'abonné, etc.

A côté de la passerelle il existe aussi le pont - simplement un autre type de passerelle, il permet d'établir la connexion entre deux réseaux locaux.

Nous nous contenterons de citer seulement d'autres termes plongés dans le bain métaphorique de route, réseau, transport d'informations. Il y en a plusieurs: voie, voie de retour, canal, routeur, etc. 


\section{MUR COUPE-FEU (ang. firewall)}

Un mur coupe-feu dans le langage informatique constitue l'équipement du serveur du réseau destiné à protéger le réseau interne contre p. ex. des utilisateurs non autorisés, des virus. Le terme d'origine nous fait penser au système de protection contre le feu, donc contre le danger dévastateur. Son calque, donc l'essai de la traduction en français, renforce encore l'image de la protection nécessaire puisque on rencontre aussi d'autres expressions-traductions comme garde-barrière, barrière de protection, écluse, mur anti-feu.

\section{CHAMPS SEMANTIQUE «NATURE»}

\section{SOURIS (ang. mouse)}

L'expression métaphorique la plus connue et probablement l'une des moins discutables soit «mouse» / la souris. Comme le raconte Gabriel Otman (Otman 1998: 357-358), son prototype a été inventé déjà en 1964 par l'ingénieur américain Douglas Engelbart sous forme «d'un boîtier en bois muni de deux disques placés à angle droit que l'on pouvait faire tourner du bout de doigt et qui déplaçaient le curseur dans l'axe vertical ou horizontal de l'écran, et d'une cordelette torsadée qui rattachait ce boîtier à l'ordinateur». L'inventeur avait reconnu dans le fil de ce boîtier la queue de souris. La métaphore n'entre en vie quotidienne qu'avec l'ordinateur Lisa d'Apple et cela en 1983. On a très vite calqué ce terme en français, mais actuellement on peut observer aussi l'emploi du vocable du registre familier mulot (petit rongeur, muridés, appelé aussi rat des champs); son histoire est intéressante aussi. La marionnette de Jacques Chirac dans l'émission télévisée satirique Les Guignols de l'info sur Canal+ l'avait employé pour la première fois, dans le contexte ironique et grâce à une grande popularité de ce programme les passionnés de l'ordinateur ont ajouté ce terme familier au vocabulaire française de l'informatique. Et bien que le dispositif nommé souris n'est plus si universel qu'avant (on le remplace par exemple par la boule de commande, «trackball»), il possède une grande famille composée de souris mécanique, souris optique, souris optomécanique, souris à capteurs rotatifs, stylo-souris, souris à ultrasons, etc. Cette dernière nous ramène à une autre métaphore du genre animalier dans le domaine de l'informatique. Souris à ultrasons c'est un dispositif émettant ses instructions sous la forme de micro ondes, la souris de ce type n'est plus liée à l'ordinateur par un fil. Le terme anglais «the Owl», donc la chouette nous fait imaginer un cyber-oiseau qui ressent et entend ce que l'homme n'est pas capable de percevoir. Otman cite Le Monde (11-12 février 1996): "The Owl (la chouette), ainsi baptisée par son fabricant, aurait tout aussi bien pu s'appeler la chauve-souris, puisqu'elle fonctionne aux ultrasons».

Quant aux métaphores «animalières», elles ne sont pas tellement nombreuses dans le vocabulaire informatique. Otman nous décrit la punaise, la puce, le virus - 
alors des termes absolument négatifs, et l'araignée qui, à la différence de ces derniers, est trouvée positive dans le monde informatique. Parlons-en en quelques mots.

\section{BOGUE (ang. bug)}

Il existe en anglais le terme «bugs» renvoyant à l'image des punaises et autres insectes qui à l'époque se collaient dans les lampes et circuits de premiers ordinateurs provoquant ainsi des courts-circuits, donc des blocages lors du fonctionnement de logiciels. Le rapport entre ce terme anglais et le terme français bogue est très étroit, bien que le français a joué sur la proximité phonétique et a créé la métaphore «végétale»: bogue c'est bien sûr l'enveloppe piquante de la châtaigne (du breton bolc'h).

\section{PUCE (ang. chip)}

L'insecte sauteur, la puce, a aidé à nommer la pastille de silicium qui contient un ensernble de circuits intégrés dont les griffes de fixation ressemblent aux pattes de puce et qui est, lui aussi, brun. Cette comparaison est totalement française, parce que le terme d'origine «chip» signifie fragment, copeau, lamelle.

\section{VIRUS}

Le virus constitue un autre exemple de la métaphore dite «animalière» - «c'est un petit programme autopropageable (donc qui est capable de se reproduire) dont la fonction est de perturber, par modification ou destruction, un système informatique dans lequel il a été installé par malveillance à l'insu de son utilisateur courant» (Otman 1998: 425). C'est alors une forme de vie ... artificielle. Un virus contamine l'ordinateur tout comme de vrais virus qui s'attaquent à un organisme vivant affaibli; il laisse ses traces, son empreinte, dans tous les programmes infectés justement comme des virus vivants en entrant dans les cellules les détruisent ou changent leurs structures. On a des virus inoffensifs, destructeurs, furtifs, dormants, mutants, encryptés, associatifs, etc. On parle de la colonie de virus, des souches du virus, mais aussi du centre de décontamination - de la clinique informatique. Les métaphores, maintenant médicales, abondent en ce point.

Il serait profitable d'y ajouter l'explication de la métaphore cheval de Troie ou Back Orifice qui est liée à celle de virus. Cheval de Troie c'est un programme introduit clandestinement dans un ordinateur, souvent sous couvert d'un programme officiel et légal, il en permet l'administration à distance, de type client / serveur, c'est-à-dire le contrôle à l'insu du légitime propriétaire.

GRAPPE (ang. cluster)

Une grappe dans le sens propre c'est «un assemblage de fleurs ou de fruits portés par des pédoncules étagés sur un axe commun» ou bien «assemblage serré de petits objets (grains, etc.), ou de personnes», dans le langage technique «groupe d'éléments réunis». Mais ici nous parlons «d'un ensemble de disques durs d'un 
serveur fonctionnant autour d'un même système électronique de manière à assurer une sécurité et des performances optimales» (Otman 1998: 152). L'image végétale très plastique a trouvé son expression aussi dans des termes comme grappe d'ordinateurs, système de stockage à grappe de disques, fonctionnement en grappe, etc.

\section{CONCLUSION}

Le présent article a été en fait une simple présentation de quelques exemples des expressions métaphoriques présentes dans le langage des utilisateurs de l'ordinateur et des net-surfeurs. Généralement nous ne nous rendons pas compte de l'impact du vocabulaire quotidien sur cette langue spécifique, appartenant au domaine de la terminologie, les langues de spécialité étant considérées en tant que le système des termes strictement techniques, précis et autoritaires. Nous y consentons absolument en soulignant notamment qu'il y a de la place, même dans le domaine étudié, celui de la terminologie informatique, pour des comparaisons intéressantes qui donnent naissance aux dénominations techniques en nous renvoyant à des éléments concrets de notre réalité quotidienne (souris, nez, courrier) ou bien à des images plus abstraits (ubiquitous computing).

\section{BIBLIOGRAPHIE, SOURCES INTERNET}

Attali. J. (1995), Les labyrinthes de l'information, Le Monde du 9 novembre 1995, p. 18; URL= http://www.synec-doc.be/doc/attali.htm

Benkirane, R., La métaphore du village global, paru lc 24.03 .1998 dans la Tribune de Genève ct sur lc site Archipress URL= http://www.archipress.org/

Eek, E. (1998), La langue française de l'informatique envisagée depuis une perspective américaine. Mcta, XLIII, 3. URL= http:/www.pum.umontreal.ca/revues/meta.htm

English, K. (1998), Une place pour la métaphore dans la théorie de la terminologie: les télécommunications en anglais et en français, URL=http://www.int-evry.fr/lfh/publications/thcse_english.htm

Hamann J. (1996), De l'ordinosaure au monde virtuel, article paru le 23 mai 1996 sur le site URL= htlp://www.ulaval.ca/scom

Huyghe, F.-B., l'Observatoire Europécn d'Infostratégie, Glossaire Internet, URL= htlp://www.strategic-road.com

Kacpszak, A. (2000), Terminologié médicale française et polonaise. Analyse formelle et sémantique, Wydawnictwo Uniwersytetu Lódzkiego, Lódź.

Lag outte, F. (1993), L'anglais de l'informatique en 1000 mots, Editions Belin. Paris.

Levine, J. R., Baroudi, C., Levine Young, M. (1998), Internet pour les nuls, $5^{\mathfrak{e}}$ edition, Sybex.

Menga, D. (2002), L'informatique se dilue dans le paysage? paru le 18/03/2002 sur le site Fondation Internet Nouvelle Génération, séminairc "Nouvelles interactions Homme-Environnement" (tenu lc 6 juin 2002) URL $=$ http://www.fing.org, http://aristote.asso.fr

Otman, G. (1998), Les mots de la cyberculture. coll. Le français retrouvé, Editions Belin, Paris.

Site URL=http://martine.gingras.net/tour/d_esprit/clairenet.html. 